\title{
Nanoscale
}

PAPER
View Article Online

View Journal | View Issue
Check for updates

Cite this: Nanoscale, 2018, 10, 17884

\section{A water-processable cellulose-based resist for advanced nanofabrication $\uparrow$}

\author{
Camilla Dore, ${ }^{a}$ Johann Osmond ${ }^{b}$ and Agustín Mihi $\mathbb{D}$ *a
}

The ideal nanofabrication technique is one that allows the mass production of high resolution submicrometric features in a cost efficient and environmentally friendly fashion. A great step towards achieving this goal has been the development of nanoimprinting lithography, a procedure with tenths of nanometres resolution while being compatible with roll-to-roll manufacturing. However, an ecofriendly resist that can be efficiently combined with this process is still missing. In this work, we demonstrate the use of hydroxypropyl cellulose (HPC) as a biocompatible, biodegradable, and water processable resist for temperature assisted nanoimprint lithography (tNIL) by fabricating different photonic architectures. The cellulose derivative is easily patterned with submicrometric features with aspect ratios greater than 1 using an elastomeric stamp and a hot plate. Silicon photonic crystals and metal nanoparticle arrays are fabricated combining cellulose with traditional nanofrabrication processes such as spincasting, reactive ion etching and metal lift off. Furthermore, advanced nanofabrication possibilities are within reach by combining the HPC with traditional resists. In particular, poly(methyl methacrylate) and HPC stacks are easily produced by liquid phase processing, where one of the two materials can be selectively removed by developing in orthogonal solvents. This capability becomes even more interesting by including nanoimprinted layers in the stack, leading to the encapsulation of arrays of air features in the resist.

Received 14th June 2018, Accepted 16th August 2018 DOI: $10.1039 / \mathrm{c} 8 \mathrm{nr} 04851 \mathrm{~g}$ rsc.li/nanoscale should rely on inexpensive, biodegradable and easily accessible materials. $^{1-3}$

Following the recent developments in the semiconductor and flexible electronics industries, among the emerging highresolution patterning techniques, nanoimprint lithography (NIL) can be considered the most promising approach for mass production of innovative optic and electronic devices. Features as small as $10 \mathrm{~nm}$ can be produced using NIL, ${ }^{4}$ and when compared to electron beam lithography (EBL) and other high-end photolithographic methods, NIL is simultaneously a low cost and more versatile technique; it is compatible with roll-to-roll technology and is suitable for large area patterning. Furthermore, in nanoimprinting lithography, resist molding is attained via mechanical deformation, making this technique suitable for patterning a broader range of materials independently of their light or electron sensitivity.

Nanoimprinting lithography is a simple and scalable technique that enables moving from the laboratory to a larger scale; however, a viable water processable ecofriendly resist for this technology is still missing. Indeed, despite the attractive features of NIL techniques, there are only a few examples of biodegradable materials used as resists for NIL, while most of the studies have aimed at developing photolithographic ${ }^{5}$ and EBL ecofriendly resists, as recently demonstrated for silk fibroin and cellulose based materials. ${ }^{6,7}$ 
NIL is a suitable method for the patterning of biopolymers, finding a wide range of applications, particularly in the production of biophotonic platforms. ${ }^{8}$ Molding of diverse cellulosic materials has previously been reported; for instance, it is possible to hot emboss liquid $\operatorname{wood}^{9}$ or to use fast imprinting methods compatible with roll-to-roll to pattern cellulose-based polymers. ${ }^{10-12}$ The feasibility of patterning these eco-friendly materials is the starting point for the development of a viable green resist for nanoimprinting lithography.

In this work, we utilize hydroxypropyl cellulose (HPC) as a thermal aided nanoimprint (tNIL) resist fully processed with water. We aim to provide the first characterization of the HPC as a green resist and demonstrate its potential by fabricating photonic crystals and 2D metal nanoparticle arrays. In doing so, we provide data on the performance of this material in common nanofabrication processes such as spincasting, reactive ion etching and metal lift off. The opportunity to use cellulose-based materials as lithographic resists is particularly appealing, first of all because cellulose is the most abundant polymer on Earth and since centuries it has been extracted from vegetal sources. In particular, hydroxypropyl cellulose is a water soluble derivative of cellulose in which some of the $\mathrm{OH}$ groups from the polysaccharide chain have been substituted with an ether group. HPC is cheap, commercially available, biodegradable and biocompatible and it is already widely used in the pharmaceutical industry as a passive drug excipient. Previous patterning attempts of water processable biopolymers such as poly vinyl alcohol (PVA) were mainly achieved in the form of free-standing membranes and by using replica molding techniques (pouring the polymer directly on the PDMS mold). PVA is frequently used only as a lift-off layer since nanoimprinting this material requires the use of hard molds under high pressures (5 MPa), which hinder conformal contact with the substrate. ${ }^{13}$ In contrast, we demonstrate herein the imprinting of HPC with extremely low pressures ( $c a$. $0.05 \mathrm{MPa}$ ) and flexible PDMS molds.

Furthermore, this cellulose based resist is not only an ecofriendly alternative, but also can be combined with traditional resists insoluble in water, leading to advanced fabrication possibilities. As an example, we fabricated alternating stacks of HPC and PMMA in which each material can be selectively removed with water or toluene, demonstrating its potential as a sacrificial layer. Polymeric multilayers are widely studied systems that find application in many different fields ranging from biotechnology ${ }^{14}$ to optics ${ }^{15}$ and that are currently employed in advanced lithographic techniques. ${ }^{16,17}$ Moreover, combining this method with tNIL, we have been able to produce ordered arrays of air-holes embedded in PMMA, opening up the path towards a straightforward nanofabrication strategy that can find application in the production of innovative optofluidic devices. ${ }^{18}$

The characterization of the cellulose-based resist begins with the fabrication of typical nanostructures by tNIL. The performance of the cellulose derivative in each lithographic step (dry etching, water lift-off and selective development) is subsequently analyzed.
The patterning process of the HPC film on silicon wafers is illustrated in Fig. 1a. Briefly, a spincast HPC film is heated at $140{ }^{\circ} \mathrm{C}$ on a hot plate above the HPC glass transition temperature while gently pressing with a pre-patterned PDMS mold. After being cooled down, the mold is released, leaving the negative pattern on the HPC layer.

\section{Results and discussion}

The first requirement for a good nanoimprinting resist is the possibility to obtain smooth and homogeneous films with tunable thicknesses via spin coating. The thickness of the film depends on the concentration of the polymer solution (which determines the solution viscosity) and on the spin-coating speed. HPC solutions in water are optically transparent and homogeneous. They are simply prepared by stirring HPC powder in deionized water (see the Experimental section). The cellulose solutions can be readily spincast on silicon wafers or glass substrates without any pre-treatment of the substrate surface. Smooth and homogeneous films of HPC with different thicknesses are obtained after spincasting. To the best of our knowledge, there is no preexisting data describing thin films of HPC obtained by spincasting from aqueous media. We provide herein a spin rate vs. thickness standard curve, recorded for different cellulose concentrations (Fig. 1b). Highly homogeneous layers of HPC on silicon were fabricated by varying the spin speed between 2000 and $5000 \mathrm{rpm}$. Lower spin rates resulted in inhomogeneous HPC films while at higher spin rates there were not considerable variations in the film thickness.

In order to characterize the surface roughness of the HPC films, a topographic analysis (Fig. 1c) of the HPC layers before and after the imprinting process was performed. We found that the root-mean-square height $(\mathrm{Sq})$ of the as prepared film was only $3 \mathrm{~nm}$, which further decreased to $0.8 \mathrm{~nm}$ after the hot embossing process using a PDMS mold.

One of the main differences between thermally assisted nanoimprinting or hot embossing and traditional photolithographic techniques is that the patterning process always leaves an excess of material between the imprinted features and the substrate. ${ }^{19}$ This residual layer must be removed in order to expose the underlying wafer and use the polymer as a lithographic mask. Good control over the thickness and homogeneity of this residual layer is required for a successful replication of the pattern in the substrate. Minimizing the residual layer thickness dramatically improves the quality of the final pattern ${ }^{20}$ minimizing the lateral erosion during the etching process.

During the embossing step of the HPC films, the polymer flows until filling the mold features after which point it stops ${ }^{21}$ and no decrease in thickness of the residual layer occurs even after prolonged embossing time. A similar behavior was observed by Lee et $a .^{22}$ during hot embossing of PMMA films on silicon using tNIL. Following a volume conserving model, it is possible to calculate a directly proportional 
a
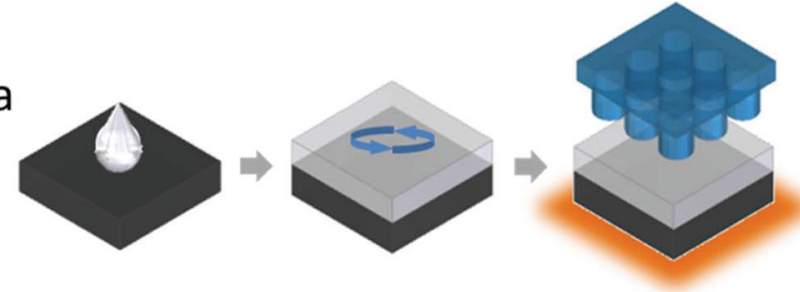

b

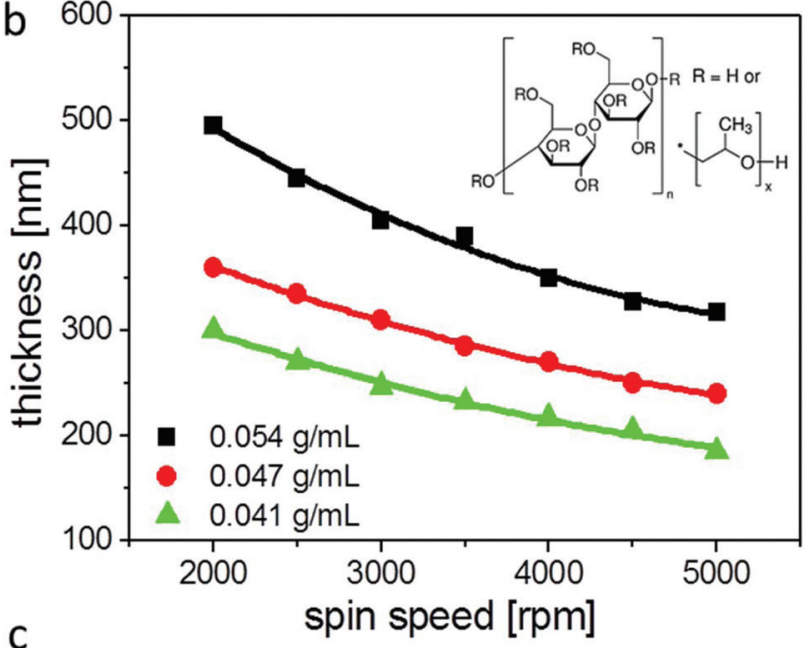

C
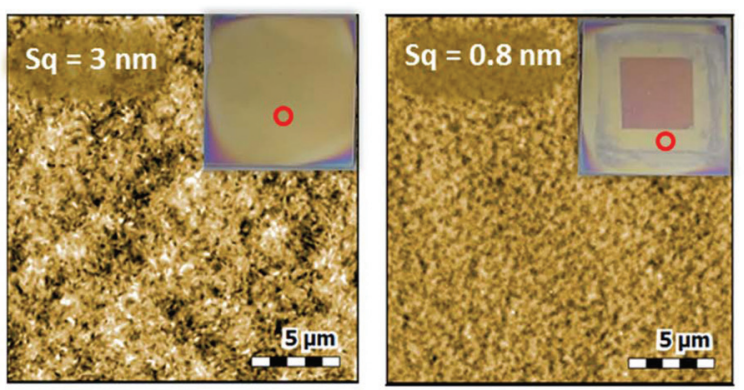
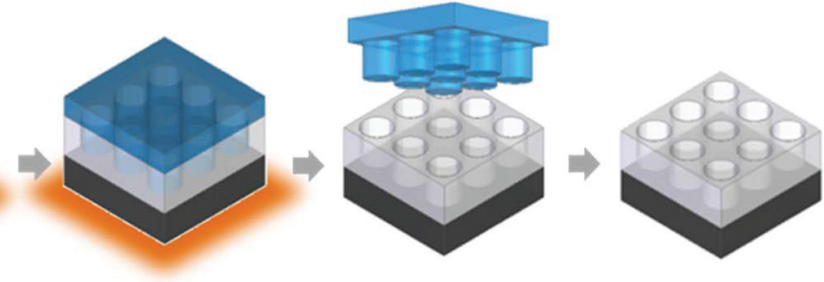

d

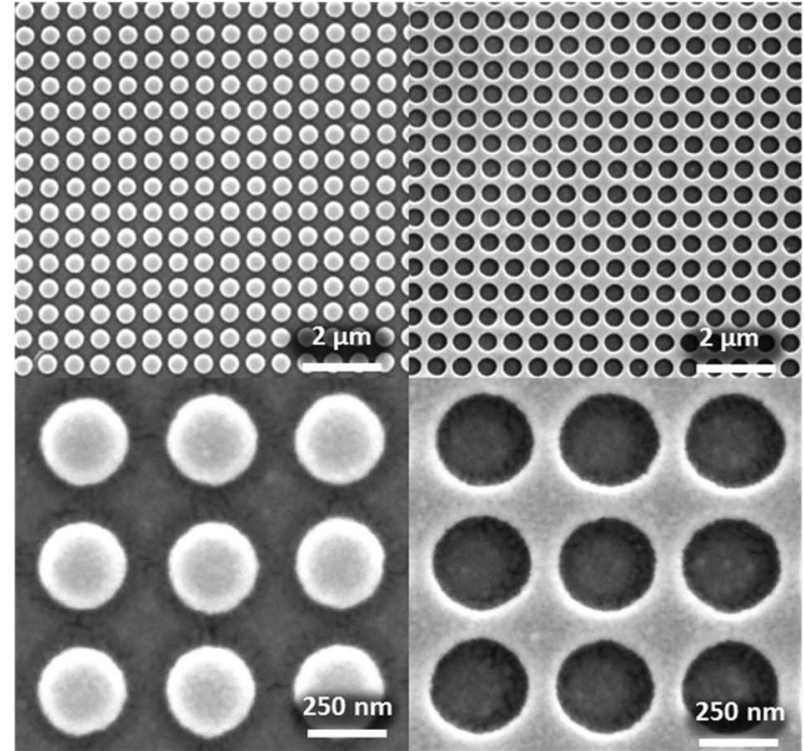

e

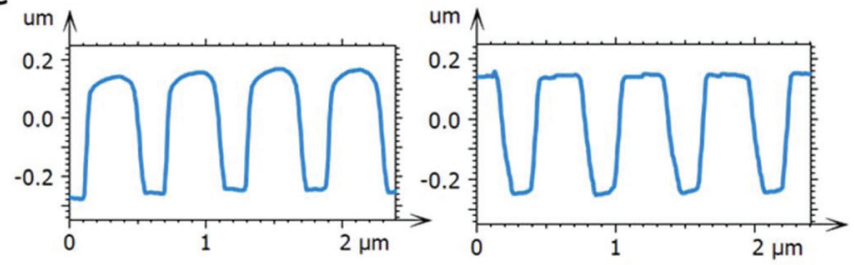

Fig. 1 (a) Schematic representation of the thermal nanoimprinting (tNIL) process employed to pattern the HPC resist on glass and silicon substrates. (b) Spin rates vs. thickness curves for HPC films spincast from aqueous solutions with concentrations of $0.054 \mathrm{~g} \mathrm{~mL}^{-1}$ (black), $0.047 \mathrm{~g} \mathrm{~mL}^{-1}$ (red) and $0.041 \mathrm{~g} \mathrm{~mL}^{-1}$ (green). (c) Atomic Force Microscopy pictures of flat HPC films as prepared (left) and after imprinting (right), reporting the measured root-mean-square height, Sq. The insets show a photograph of the samples before and after imprinting, in which the red dot highlights the scanned area. (d) Exemplar SEM top view image of the pillars (left) and holes (right) of an imprinted hydroxypropyl cellulose film on silicon at different magnifications (samples have been covered with $10 \mathrm{~nm}$ of gold). (e) Atomic Force Microscopy cross sectional profiles of the imprinted features in (d).

relationship between residual layer thickness and the initial film thickness that depends only on the mold pattern geometry.

The excellent mechanical stability of HPC enabled the fabrication of pillar and hole arrays with aspect ratios greater than 1 and with minimum feature size down to $100 \mathrm{~nm}$ (Fig. 1d, e and 2f). Alternatively, HPC can also be patterned via electron beam lithography (EBL) with analogous submicrometre resolution. Dosage tests and further details of the performance of HPC under electron beam exposure are provided in the ESI. $\dagger$

Large area patterns of $1 \mathrm{~cm}^{2}$ were produced with tNIL in cellulose showing excellent replication of the PDMS pattern. Furthermore, hot embossing of HPC does not require any surface treatment of the PDMS mold and it can be performed at relatively low temperatures $\left(140{ }^{\circ} \mathrm{C}\right)$ gently pressing the mold against the film for 1 minute.

At this point of the process, a patterned hydroxypropyl cellulose layer is left on top of a silicon wafer. To transfer the pattern from the resist to the underlying substrate, dry etching processes are typically applied. Reactive Ion Etching (RIE) is one of the most advanced techniques suitable for large scale integration in surface micromachining processes. However, the production of high aspect ratio features (deep etching) requires a fine-tuning of the process parameters and an adequate choice of precursor gases to obtain anisotropic etching.

Silicon deep etching processes often rely on chemistries that protect the side walls to ensure minimum lateral erosion and produce vertical side walls and sharp features. ${ }^{23}$ Among these techniques, the mixed mode Bosch process (pseudo 

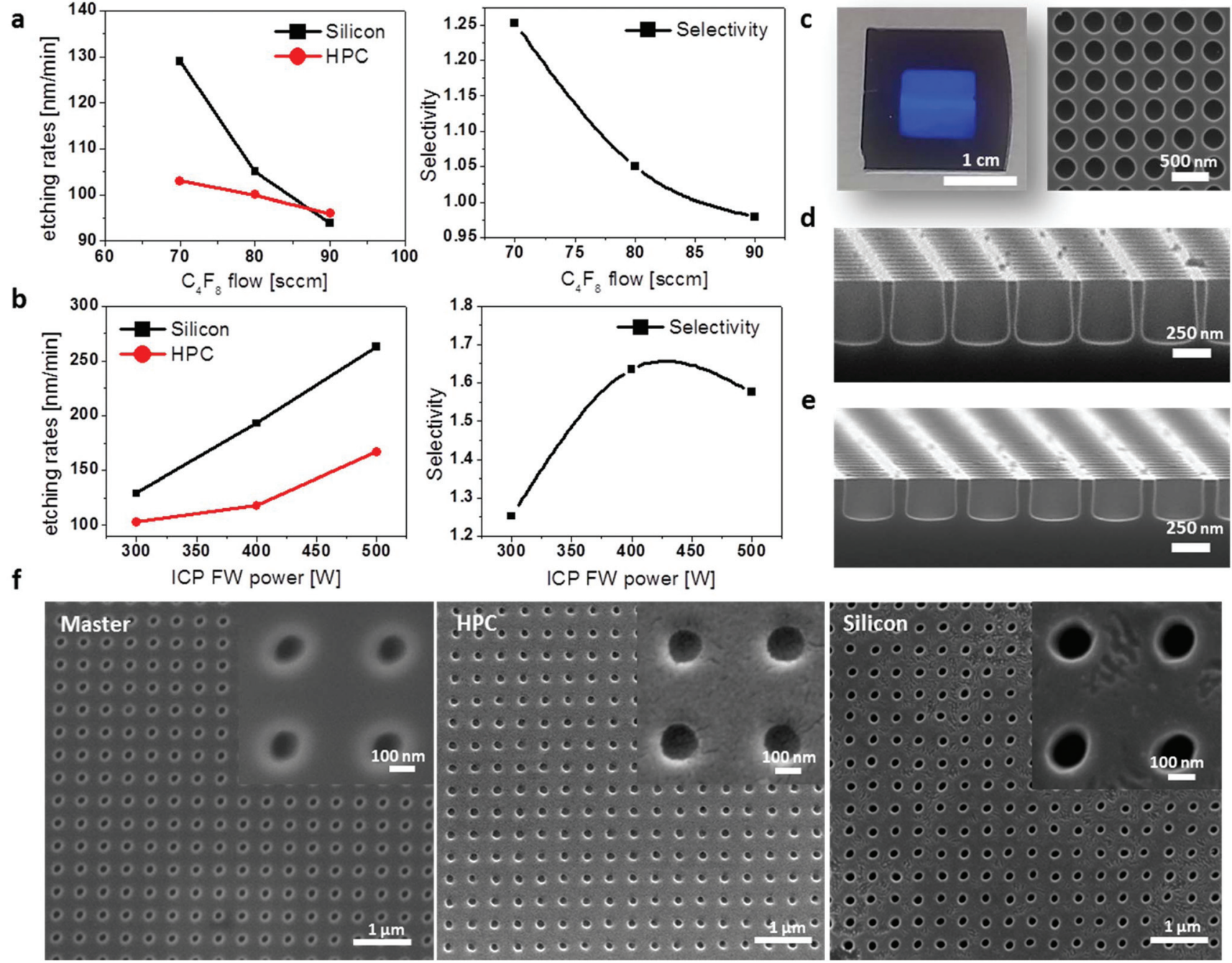

Fig. 2 (a) Etching rates of silicon (black) and HPC (red) and the calculated selectivity of the process for $\mathrm{C}_{4} \mathrm{~F}_{8}$ flows equal to 70,80 and 90 sccm with a fixed ICP power of $300 \mathrm{~W}$. (b) Etching rates of silicon (black) and HPC (red) and the calculated selectivity of the process for ICP powers equal to 300,400 and $500 \mathrm{~W}$ with a $\mathrm{C}_{4} \mathrm{~F}_{8}$ flow of $70 \mathrm{sccm}$. (c) Picture and top view SEM image of a patterned silicon substrate after RIE. (d) SEM cross section of a sample etched for $2 \mathrm{~min}$ with $70 \mathrm{sccm} \mathrm{C} \mathrm{F}_{8}$ flow and $500 \mathrm{~W}$ ICP power. Hole depth corresponds to $410 \mathrm{~nm}$. (e) SEM cross section of a sample etched for $3 \mathrm{~min}$ with $90 \mathrm{sccm} \mathrm{C} \mathrm{F}_{8}$ flow and $300 \mathrm{~W}$ ICP power. Holes depth corresponds to $260 \mathrm{~nm}$. (f) $120 \mathrm{~nm}$ diameter hole patterns transferred to silicon using HPC as a resist. From left to right: SEM top view images of the original master, imprinted HPC and silicon substrate after RIE.

Bosch) used in this work is particularly suitable for nanoscale etching $^{24,25}$ since it allows us to obtain smooth sidewalls without the need for cryogenic cooling. For the RIE conditions used in this study (see the Experimental section), we investigated the etching rate of HPC and its selectivity versus silicon. The ratio between the etching rate of the resist and the wafer substrate determines the maximum possible depth of the etched features that can be transferred to the substrate. Mask erosion can thus be an extremely limiting factor in dry etching processes, particularly when a polymeric resist is employed.

The imprinted HPC films on silicon wafers underwent different RIE steps, which were performed in order to determine the effect of the RIE conditions on the replication of the pattern. Setting the optimal etching parameters usually requires several sets of experiments and is far beyond the purpose of this paper. Here we report a preliminary study of the etching behavior of hydroxypropyl cellulose in a DRIE process as a general guideline that can be subjected to further optimizations. In general, all the etching conditions studied herein yielded a faithful replication of the pattern into the silicon wafer. Optical and electron microscopic inspections indicated excellent homogeneity along the patterned area and the samples showed the characteristic iridescent color of photonic crystals (Fig. 2c). The minimum feature size tested in this work is $100 \mathrm{~nm}$ as illustrated in the ESI† (Fig. S10) and was only limited by the lack of pre-patterned molds with smaller features in our laboratory. We have not observed any increase in roughness or distortion of the features in any of 
the patterned HPC films; hence, we believe that smaller features can be achieved in HPC if new composite stamps with nanometric patterns are utilized. ${ }^{26}$

Fig. $2 \mathrm{a}$ and $\mathrm{b}$ show the etching rates of silicon and HPC and the corresponding selectivity obtained for three different $\mathrm{C}_{4} \mathrm{~F}_{8}$ flows at a fixed ICP power of $300 \mathrm{~W}$ and for three different ICP powers at a fixed $\mathrm{C}_{4} \mathrm{~F}_{8}$ flow of $70 \mathrm{sccm}$. The maximum selectivity is obtained for an ICP power of $400 \mathrm{~W}$ with a $\mathrm{C}_{4} \mathrm{~F}_{8}$ flow of $70 \mathrm{sccm}$ and corresponds to a value of 1.64, which is comparable with the values reported for PMMA ( $c a$. 2). ${ }^{27-29}$ Fig. 2d and e show SEM cross sections of two samples etched under different RIE conditions. It is worth noting that increasing the ICP power value with $\mathrm{a}_{4} \mathrm{~F}_{8}$ flow fixed at 300 sccm deteriorates the degree of anisotropy of the process. To compensate for this effect one possibility is to increase the $\mathrm{C}_{4} \mathrm{~F}_{8}$ flow (Fig. 2e); however, this deteriorates the selectivity of the process.

The poor selectivity of resist masks is well known to the nanofabrication community, which restricts their use to low aspect ratio features. When deep etching is required, photoresist masks are an intermediate step towards the deposition of a more robust material such as metals. In these cases, a metal is evaporated on top of the resist mask, and then the resist is removed from the wafer, leaving the desired metallic pattern behind. The removal of the resist mask that prevented the metal deposition on the silicon wafer, typically called "liftoff", is a commonly used nanofabrication step that is most necessary to obtain well-defined features. The possibility to employ the lift-off technique using HPC as a sacrificial layer is particularly attractive ${ }^{5,6}$ considering that the whole process can be implemented using only water as a solvent, therefore minimizing the use and generation of hazardous and polluting wastes.

To demonstrate the validity of the cellulose resist in a liftoff process with water, an array of aluminium nanoparticles organized in a square array were fabricated. A schematic of the fabrication process is depicted in Fig. 3c: briefly, HPC was spincast on glass substrates and thermally imprinted, followed by a short RIE step required to remove the residual layer from the imprinted HPC structures and to expose the glass substrate underneath. Next, $150 \mathrm{~nm}$ of aluminum are thermally evaporated above the sample. Finally, the HPC sacrificial layer is removed in water. After this lift-off step, only an array of aluminum nanoparticles remains on the glass surface.

Nanoparticle square arrays with lattice parameters of 400 , 500 and $600 \mathrm{~nm}$ were fabricated using HPC as a sacrificial layer. A representative SEM picture of the $400 \mathrm{~nm}$ lattice parameter nanoparticle array is shown in Fig. 3a. The optical characterization of the samples (Fig. 3b) shows the presence of an intense extinction peak corresponding to the Bragg surface plasmon polariton (Bragg-SPP), which red-shifts with increasing lattice parameter, which evidences the high quality of the plasmonic crystals fabricated. Further tuning of the nanoparticle diameter can be achieved by varying the duration of the etching step that precedes the metal deposition $(\mathrm{ESI} \dagger)$.

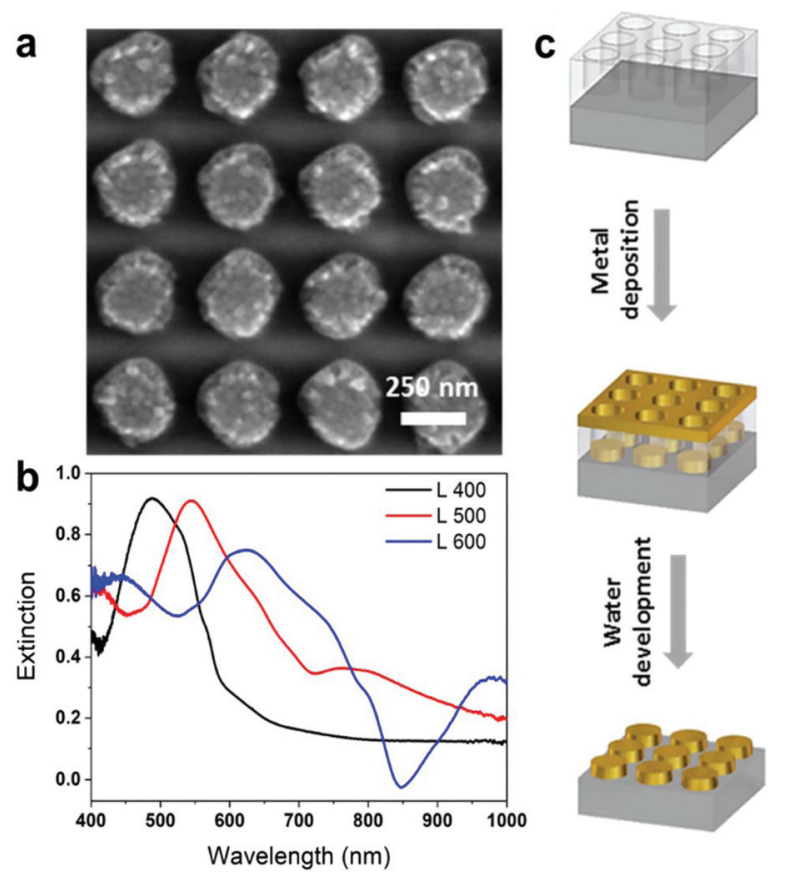

Fig. 3 (a) Top view SEM of the fabricated square array of aluminium pillars with $300 \mathrm{~nm}$ diameter, $150 \mathrm{~nm}$ height and $400 \mathrm{~nm}$ lattice parameter. (b) Extinction spectra of the metal arrays with varying lattice parameters of $400 \mathrm{~nm}$ (black), $500 \mathrm{~nm}$ (red) and $600 \mathrm{~nm}$ (blue). (c) Schematic of the lift-off process employed to fabricate aluminium nanoparticle arrays on glass substrates.

We have demonstrated so far the viability of hydroxypropyl cellulose as an environmentally friendly water processable resist, providing spin rates, etching selectivity values and lift off procedures. It is worth noting that the type of cellulose derivative and its substitution degree might affect the performance of the polymer under chemical etching and water development. Therefore, it might be possible to find derivatives with improved response under the nanofabrication processes.

In the following sections, we will demonstrate that HPC is not only a water processable resist but can also yield new and exciting advanced nanofabrication possibilities. HPC is biocompatible and is completely processed in water; thus it can be used as a means to pattern many biological materials incompatible with previous corrosive reagents. Furthermore, water is orthogonal to most solvents used with traditional resists, meaning that HCP can be combined with them via liquid-phase processing and selectively removed. To demonstrate this additional functionality, we fabricated HPC and poly(methyl methacrylate) (PMMA) multilayers by alternate deposition of both polymers. $\mathrm{ABAB}$ and BABA stacks were fabricated by alternate spincasting of A (HPC solutions in water) and B (PMMA solutions in Toluene). Considering that HPC is not soluble in toluene and $\mathrm{PMMA}^{30}$ is not soluble in water, it is possible to employ both orthogonal solvents to specifically dissolve a particular polymer in the stack.

Fig. 4 shows cross-sectional SEM images of the ABAB and BABA multilayer stacks prior to and after removal of the A or $\mathrm{B}$ 


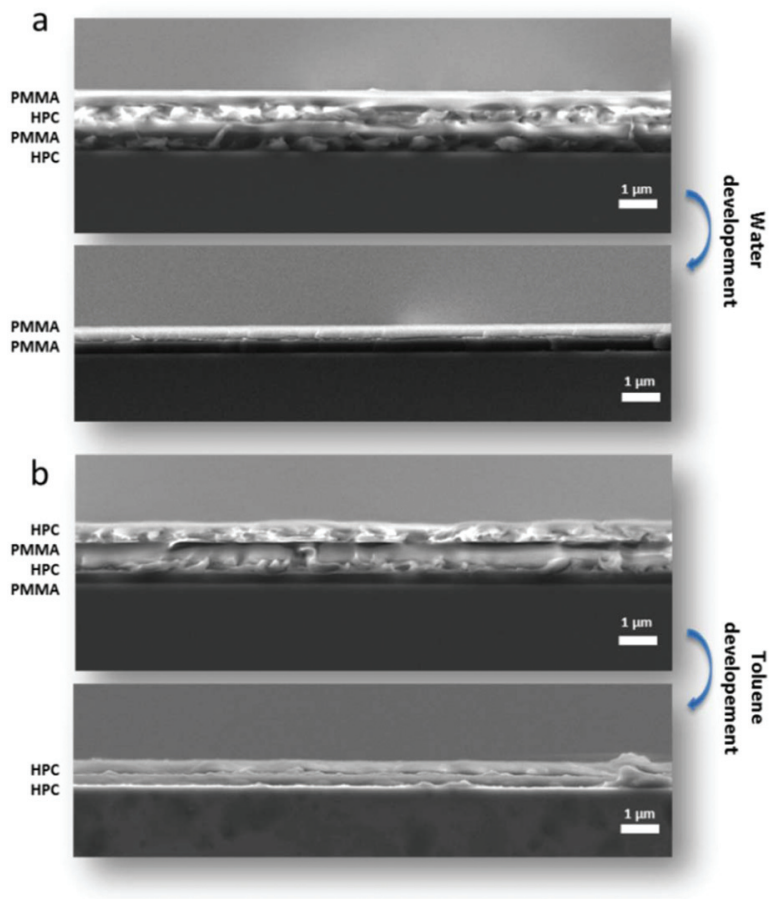

Fig. 4 Cross sectional SEM images of polymeric stacks on silicon substrates. The film thickness is $450 \pm 50 \mathrm{~nm}$ for both HPC and PMMA layers. (a) HPC-PMMA-HPC-PMMA four layer stacks before and after development in water (HPC layer dissolution). (b) PMMA-HPC-PMMA-HPC four layer stack before and after devolvement in toluene (PMMA layer dissolution). layers. As can be clearly seen from the picture, depending on the solvent used for the development it is possible to completely dissolve one or the other material, obtaining a monocomponent system consisting of two layers separated by a thin film of air. It is worth noting that if the films are developed carefully it is possible to avoid the lift-off of the upper layer and the remaining undeveloped films adhere to the substrate and collapse onto each other. Since we believe that water diffuses laterally from the edges of the film towards the center, dissolving the HPC can lead to delamination if thick layers of HPC are employed. Using submicrometric HPC films minimized the cracking of the PMMA layers and facilitated stack fabrication.

This can be exploited to fabricate novel architectures with embedded air cavities for instance. To illustrate this advanced nanofabrication possibility, we combined thermal nanoimprint lithography with multilayer stacks of PMMA and HPC to fabricate a 2D air cavity array embedded in PMMA (Fig. 5a).

To fabricate this challenging architecture, a $300 \mathrm{~nm}$ layer of PMMA is spin coated on a silicon substrate and thermally imprinted as previously described. Next, HPC and PMMA are sequentially spin coated on the tNIL-PMMA layer: first a thin layer of HPC followed by a second layer of PMMA at the top of the stack. The development of the stack with water dissolves the thin cellulose layer and the top PMMA layer collapses onto the bottom one, encapsulating the nanoimprinted array of holes. Fig. 5 shows cross sectional SEM images of the tNIL-PMMA/HPC/PMMA stack before and after development with water of the HPC. The presence of air cavities in the film of PMMA is further confirmed by changes in the reflectance spectra of the stacks (Fig. 5c) and by optical inspection of the

a

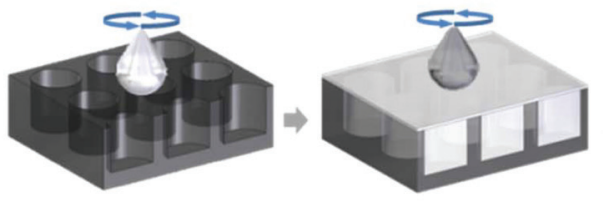

b
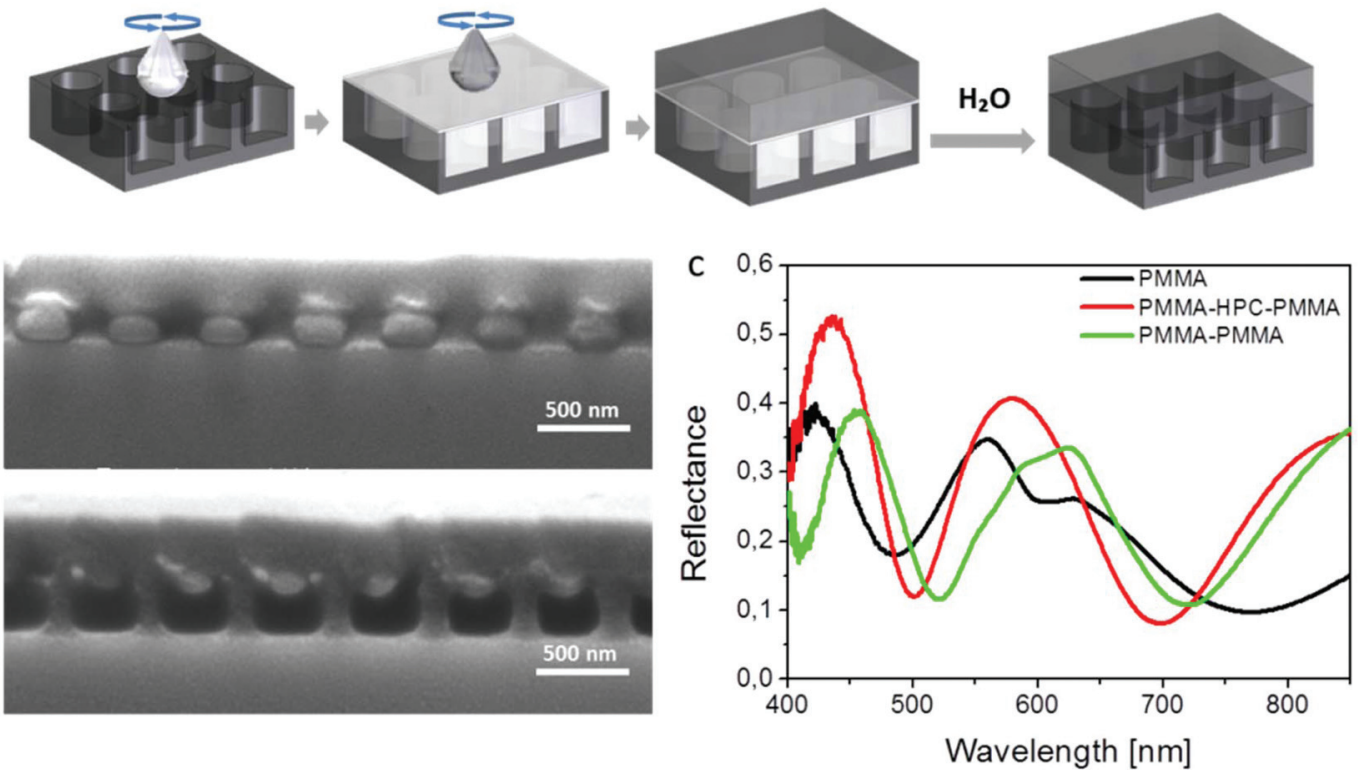

Fig. 5 (a) Schematic process employed to fabricate periodic air cavity arrays embedded in PMMA: briefly, HPC is spincast on thermally imprinted PMMA (tNIL_PMMA), and then a layer of PMMA is spincasted on top of the stack. Finally, the HPC is removed with water, leaving a hole-array embedded in PMMA. (b) Cross sectional SEM images of imprinted PMMA-HPC-PMMA stacks on silicon substrates before (top) and after (bottom) development in water. (c) FTIR reflection spectra of imprinted PMMA on silicon (black) and imprinted PMMA-HPC-PMMA stacks on silicon substrates before (red) and after (green) development in water. 
films with the naked eye. The tNIL-PMMA film appears iridescent due to the $500 \mathrm{~nm}$ lattice parameter 2D grating. The specular reflectance spectrum from tNIL-PMMA (Fig. 5c, black line) presents the characteristic Fabry-Perot oscillations originating from the thin film, together with presenting features in the 550 and $700 \mathrm{~nm}$ wavelength ranges, indicating the existence of diffraction.

When the holes in the stack are filled with HPC, the HCP and PMMA layers are index matched (refractive indexes are 1.49 for both materials), the iridescence of the pattern disappears and no distinctive features are present in the reflection spectra (red line). After developing HPC, the presence of periodic air voids imbedded in the PMMA returns the iridescent aspect of the sample and diffraction features appear again in the reflection spectrum in the 550-700 $\mathrm{nm}$ range.

\section{Conclusions}

We have demonstrated the use of hydroxypropyl cellulose as a water processable resist. Patterned areas of $1 \mathrm{~cm}^{2}$ with submicrometric features and aspect ratios higher than 1 were fabricated in silicon using a thermally assisted nanoimprinting technique. We provide key values for spincasting rates, dry etching recipes and selectivity values of the cellulose resist comparable with the data reported in the literature for PMMA. The fabrication of metal nanostructures is also possible by combining metal deposition on the imprinted resist followed by water removal of the cellulose derivative. High quality plasmonic crystals with varying lattice parameter were fabricated and their optical response was characterized by optical spectroscopy. The cellulose based resist has similar characteristics to traditional resists but is biocompatible and is completely processed with water, opening up possibilities for nanostructuring many biological materials. Furthermore, HPC can be used in combination with PMMA, both resists being processed with orthogonal solvents, enabling many advanced nanofabrication possibilities. To illustrate this added functionality, we fabricated multilayer stacks of PMMA and HPC in which one of the two materials can be selectively removed by just developing the stack in water or toluene. In sum, hydroxypropyl cellulose is a green and water processable nanoimprinting resist compatible with mass production processes. HPC is processed orthogonally to most common resists; hence it can be combined with them to yield novel nanostructures with potential application in photonics, microfluidics and advanced lithographic techniques.

\section{Experimental}

\section{Materials}

Prepatterned silicon masters were purchased from Cemitec (Navarra, Spain) and from EULITHA (Switzerland). A hard olydimetylsiloxane (PDMS) silicone elastomer kit was purchased from Gelest (Morrisville, PA 19067, USA) and a soft PDMS
Sylgard184 silicone elastomer kit from Dow Corning Corporation (Auburn, MI 48611, USA). $1 \mathrm{H}, 1 \mathrm{H}, 2 \mathrm{H}, 2 \mathrm{H}$ Perfluorooctyltrichlorosilane (PFOTS), 97\%, was purchased form Alfa Aesar (Thermo Fisher GmbH, Karlsruhe, Germany). Hydroxypropyl cellulose (HPC), with average $M_{\mathrm{w}} \sim 100000$, powder, 20 mesh particle size (99\% through) and degree of substitution DS $=3$, and poly(methylmethacrylate) (PMMA), average $M_{\mathrm{w}} \sim 15000$, were purchased from Sigma-Aldrich Quimica SL (Madrid, Spain).

\section{PDMS molds}

PDMS molds were fabricated following previously reported methods. ${ }^{31}$ Briefly, silicon masters were functionalized with PFOTS (perfluorooctyltrichlorosilane) as an anti-sticking layer, lowering the surface energy and allowing the easy release of cured PDMS. The molds were fabricated casting on the master first a thin layer of hard-PDMS and a second thick layer of soft PDMS as supports. The choice of using a composite mold is necessary in order to achieve simultaneously mechanical stability of the pattern and conformal contact with the substrate.

The three $1 \mathrm{~cm}^{2}$ patterns used in this work consist of square arrays of cylindrical pillars with $300 \mathrm{~nm}$ diameter, $350 \mathrm{~nm}$ height and lattice parameters of 400, 500 and $600 \mathrm{~nm}$.

\section{HPC films}

First, a stock solution of HPC powder in water with a concentration of $217 \mathrm{mg} \mathrm{mL}^{-1}$ was prepared by mixing and intensively stirring the HPC powder in DI water. This stock solution was further diluted in water in order to obtain three different aqueous solutions of 41,47 and $54 \mathrm{mg} \mathrm{mL}$. Each of the aqueous solutions was stirred on a magnetic plate for $3 \mathrm{~h}$. The HPC solutions were directly spin coated on clean silicon and glass surfaces. The spin time was 1 minute and the spin acceleration $1000 \mathrm{rpm} \mathrm{s}^{-1}$ for all the experiments performed. Thin films of HPC with thickness ranging between $2 \mu \mathrm{m}$ and $50 \mathrm{~nm}$ were obtained by changing the HPC solution concentrations and the speed of spin coating.

\section{Thermal nanoimprint lithography}

HPC films deposited on silicon were placed on a hot plate at $140{ }^{\circ} \mathrm{C}$ (glass transition temperature of HPC is $105^{\circ} \mathrm{C}^{32}$ ). Next, a patterned PDMS mold was gently pressed against the substrate for 1 minute. Samples were left to cool down to room temperature and finally demolded.

\section{Sample characterization}

Flat HPC film thicknesses were obtained by the numerical fitting of the Fabry-Perot oscillations from the experimental reflection spectra using the transfer matrix formalism. The HPC refractive index value was reported in previous studies. ${ }^{33}$ The reflectivity and transmission of the samples were measured using a Fourier Transform Infrared Spectrometer (FTIR, Bruker Vertex 70) attached to a microscope with a $4 \times$ objective. The depth of the features in HPC and silicon patterns was measured using a Keysight 5100 scanning probe microscope (AFM tapping mode configuration) and scanning 
electron microscopy (SEM QUANTA FEI 200 FEG-ESEM) cross sectional image analysis.

\section{Dry etching conditions}

Samples were etched using an Oxford Instruments Plasmalab System 100 ICP. Selectivity values were calculated from five different sets of etching conditions: (i) $300 \mathrm{~W}$ ICP Fw power and $70 \mathrm{sccm} \mathrm{C}_{4} \mathrm{~F}_{8}$ flow, (ii) $300 \mathrm{~W}$ ICP Fw power and $80 \mathrm{sccm}$ $\mathrm{C}_{4} \mathrm{~F}_{8}$ flow, (iii) $300 \mathrm{~W}$ ICP Fw power and $90 \mathrm{sccm} \mathrm{C}_{4} \mathrm{~F}_{8}$ flow, (iv) $400 \mathrm{~W}$ ICP Fw power and $70 \mathrm{sccm} \mathrm{C}_{4} \mathrm{~F}_{8}$ flow, and (v) $300 \mathrm{~W}$ ICP Fw power and $90 \mathrm{sccm} \mathrm{C}_{4} \mathrm{~F}_{8}$ flow. In all the processes, the pressure has been set to $15 \mathrm{mTorr}$, the RF generator power to $35 \mathrm{~W}$ and the $\mathrm{SF}_{6}$ gas flow to $45 \mathrm{sccm}$. Silicon and HPC etching rates were calculated by linear fitting of the etching depth (see ESI $\dagger$ ) for 1, 2 and 3 minutes etching time (samples i, ii, and iii) and for etching times 1 and 2 minutes (iv, v).

\section{Metal deposition and lift off}

Aluminum was deposited via e-beam evaporation (AJA International Inc. ATC-8E Orion). Samples were developed in running DI water for 30 seconds followed by 20 minutes sonication in DI $\mathrm{H}_{2} \mathrm{O}$.

PMMA-HPC multilayer stacks: HPC and PMMA stacks were fabricated by alternate spin coating at $3000 \mathrm{rpm}$ an HPC solution in DI water $\left(0.054 \mathrm{~g} \mathrm{~mL}^{-1}\right)$ and a solution of PMMA in toluene $(10 \mathrm{wt} \%)$ followed by drying at $50{ }^{\circ} \mathrm{C}$ on a hot plate for 15 min. HPC (PMMA) layers were developed by immersing the samples in water (toluene) for 10 minutes. The samples were dried in air.

Imprinted multilayer stacks: first, a thin layer of PMMA (10 wt $\%, w=3000 \mathrm{rpm}$ ) was hot embossed at $90{ }^{\circ} \mathrm{C}$. Second, a dispersion of HPC in water $\left(0.0165 \mathrm{~g} \mathrm{~mL}^{-1}\right)$ was spin coated at $3000 \mathrm{rpm}$ on top of the imprinted PMMA. Finally, a layer of PMMA was deposited using the same conditions as for the first PMMA layer. Samples were developed in water for 10 minutes and dried in air.

\section{Conflicts of interest}

There are no conflicts to declare.

\section{Acknowledgements}

The Spanish Ministerio de Economía, Industria y Competitividad (MINECO) is gratefully acknowledged for its support through Grant No. MAT2016-79053-P and through Grant No. SEV-2015-0496 in the framework of the Spanish Severo Ochoa Centre of Excellence program. AM was funded by a Ramón y Cajal fellowship (RYC-2014-16444). This project has received funding from the European Research Council (ERC) under the European Union's Horizon 2020 research and innovation program (Grant Agreement No. 637116, ENLIGHTMENT). The authors would like to acknowledge M. Simón and A. Gómez for AFM measurements.

\section{References}

1 A. M. Pandele, P. Neacsu, A. Cimpean, A. I. Staras, F. Miculescu, A. Iordache, S. I. Voicu, V. K. Thakur and O. D. Toader, Appl. Surf. Sci., 2018, 438, 2.

2 D. Trache, M. H. Hussin, M. K. M. Haafiz and V. K. Thakur, Nanoscale, 2017, 9, 1763.

3 S. I. Voicu, R. M. Condruz, V. Mitran, A. Cimpean, F. Miculescu, C. Andronescu, M. Miculescu and V. K. Thakur, ACS Sustainable Chem. Eng., 2015, 4, 1765.

4 S. Y. Chou and P. R. Krauss, Imprint lithography with sub$10 \mathrm{~nm}$ feature size and high throughput, Microelectron. Eng., 1997, 35, 237-240, DOI: 10.1016/S0167-9317(96) 00097-4.

5 V. Linder, B. D. Gates, D. Ryan, B. A. Parviz and G. M. Whitesides, Water-soluble sacrificial layers for surface micromachining, Small, 2005, 1, 730-736, DOI: 10.1002/smll.200400159.

6 S. Kim, B. Marelli, M. A. Brenckle, A. N. Mitropoulos, E.-S. Gil, K. Tsioris, H. Tao, D. L. Kaplan and F. G. Omenetto, All-water-based electron-beam lithography using silk as a resist, Nat. Nanotechnol., 2014, 9, 306-310, DOI: $10.1038 /$ NNANO.2014.47.

7 S. Takei, H. Maki, K. Sugahara, K. Ito and M. Hanabata, Inedible cellulose-based biomass resist material amenable to water-based processing for use in electron beam lithography, AIP Adv., 2015, 5, 77141, DOI: 10.1063/1.4927210.

8 J. J. Amsden, P. Domachuk, A. Gopinath, R. D. White, L. D. Negro, D. L. Kaplan and F. G. Omenetto, Rapid nanoimprinting of silk fibroin films for biophotonic applications, Adv. Mater., 2010, 22, 1746-1749, DOI: 10.1002/ adma.200903166.

9 M. Worgull, M. Schneider, M. Röhrig, T. Meier, M. Heilig, A. Kolew, K. Feit, H. Hölscher and J. Leuthold, Hot embossing and thermoforming of biodegradable three-dimensional wood structures, RSC Adv., 2013, 3, 20060, DOI: 10.1039/C3RA42642D.

10 T. Mäkelä, M. Kainlauri, P. Willberg-Keyriläinen, T. Tammelin and U. Forsström, Fabrication of micropillars on nanocellulose films using a roll-to-roll nanoimprinting method, Microelectron. Eng., 2016, 163, 1-6, DOI: 10.1016/j. mee.2016.05.023.

11 T. Mäkelä, T. Haatainen and J. Ahopelto, Roll-to-roll printed gratings in cellulose acetate web using novel nanoimprinting device, Microelectron. Eng., 2011, 88, 20452047, DOI: 10.1016/j.mee.2011.02.016.

12 A. Espinha, C. Dore, C. Matricardi, M. I. Alonso, A. R. Goñi and A. Mihi, Hydroxypropyl cellulose photonic architectures by soft nanoimprinting lithography, Nat. Photonics, 2018, 8, 13131, DOI: 10.1038/s41566-018-0152-1.

13 K.-ichiro Nakamatsu, K. Tone and S. Matsui, Nanoimprint and Lift-Off Process Using Poly(vinyl alcohol), Jpn. J. Appl. Phys., 2005, 44(11), 8186-8188.

14 S. de Koker, R. Hoogenboom and B. G. de Geest, Polymeric multilayer capsules for drug delivery, Chem. Soc. Rev., 2012, 41, 2867-2884, DOI: 10.1039/C2CS15296G. 
15 Z. Wang, J. Zhang, J. Xie, Z. Wang, Y. Yin, J. Li, Y. Li, S. Liang, L. Zhang, L. Cui, H. Zhang and B. Yang, Polymer Bragg stack as color tunable photonic paper, J. Mater. Chem., 2012, 22, 7887, DOI: 10.1039/C2JM15943K.

16 C. Wang and S. Y. Chou, Integration of Metallic Nanostructures in Fluidic Channels for Fluorescence and Raman Enhancement by Nanoimprint Lithography and Lift-off on Compositional Resist Stack, Microelectron. Eng., 2012, 98, 693-697, DOI: 10.1016/j.mee.2012.05.051.

17 Y. Chen, Nanofabrication by electron beam lithography and its applications, Microelectron. Eng., 2015, 135, 57-72, DOI: 10.1016/j.mee.2015.02.042.

18 H. Andagana and X. A. Cao, Nanofabrication of photonic crystal slabs with sealed airholes for optofluidic applications, Microelectron. Eng., 2014, 114, 17-21, DOI: 10.1016/ j.mee.2013.09.005.

19 J. A. Rogers and H. H. Lee, Unconventional nanopatterning techniques and applications, Wiley-Blackwell, Oxford, 2009.

20 K.-d. Kim, J.-h. Jeong, Y.-s. Sim and E.-s. Lee, Minimization of residual layer thickness by using the optimized dispensing method in S-FILTM process, Microelectron. Eng., 2006, 83, 847-850, DOI: 10.1016/j.mee.2006.01.037.

21 H. Schift and A. Kristensen, in Springer Handbook of Nanotechnology, ed. B. Bhushan, Springer Berlin Heidelberg, Berlin, Heidelberg, 2010, pp. 271-312.

22 H.-J. Lee, H. W. Ro, C. L. Soles, R. L. Jones, E. K. Lin, W.-l. Wu and D. R. Hines, Effect of initial resist thickness on residual layer thickness of nanoimprinted structures, J. Vac. Sci. Technol., B: Microelectron. Nanometer Struct.-Process., Meas., Phenom., 2005, 23, 3023, DOI: 10.1116/1.2101776.

23 H. V. Jansen, M. J. de Boer, S. Unnikrishnan, M. C. Louwerse and M. C. Elwenspoek, Black silicon method, J. Micromech. Microeng., 2009, 19, 33001, DOI: 10.1088/0960-1317/19/3/033001.

24 S. S. Walavalkar, C. E. Hofmann, A. P. Homyk, M. D. Henry, H. A. Atwater and A. Scherer, Tunable visible and near-IR emission from sub-10 $\mathrm{nm}$ etched single-crystal Si nanopillars, Nano Lett., 2010, 10, 4423-4428, DOI: 10.1021/ nl102140k.

25 M. D. Henry, S. Walavalkar, A. Homyk and A. Scherer, Alumina etch masks for fabrication of high-aspect-ratio silicon micropillars and nanopillars, Nanotechnology, 2009, 20, 255305, DOI: 10.1088/0957-4484/20/25/255305.

26 M. A. Verschuuren, M. Megens, Y. Ni, H. van Sprang and A. Polman, Large area nanoimprint by substrate conformal imprint lithography (SCIL), Adv. Opt. Technol., 2017, 6, 9554, DOI: 10.1515/aot-2017-0022.

27 M. D. Henry, S. Walavalkar, A. Homyk, M. D. Henry, A. Scherer and C. D. O. Silicon, ICP etching of silicon for micro and nanoscale devices, 2010.

28 A. Goodyear, M. Boettcher, I. Stolberg and M. Cooke, in Direct comparison of the performance of commonly used e-beam resists during nano-scale plasma etching of $\mathrm{Si}, \mathrm{SiO}_{2}$, and $\mathrm{Cr}$, ed. Q. Lin, S. U. Engelmann and Y. Zhang, SPIE, 2015, p. 94280V.

29 J. Kim, D. C. Joy and S.-Y. Lee, Controlling resist thickness and etch depth for fabrication of 3D structures in electronbeam grayscale lithography, Microelectron. Eng., 2007, 84, 2859-2864, DOI: 10.1016/j.mee.2007.02.015.

30 C. B. Walsh and E. I. Franses, Ultrathin PMMA films spincoated from toluene solutions, Thin Solid Films, 2003, 429, 71-76, DOI: 10.1016/S0040-6090(03)00031-2.

31 T. W. Odom, J. C. Love, D. B. Wolfe, K. E. Paul and G. M. Whitesides, Improved Pattern Transfer in Soft Lithography Using Composite Stamps, Langmuir, 2002, 18, 5314-5320, DOI: 10.1021/la020169l.

32 A. Gómez-Carracedo, C. Alvarez-Lorenzo, J. L. GómezAmoza and A. Concheiro, J. Therm. Anal. Calorim., 2003, 73, 587-596, DOI: 10.1023/A:1025434314396.

33 P. Molet, J. L. Garcia-Pomar, C. Matricardi, M. Garriga, M. I. Alonso and A. Mihi, Ultrathin Semiconductor Superabsorbers from the Visible to the Near-Infrared, $A d v$. Mater., 2018, 30(9), 1705876, DOI: 10.1002/adma.201705876. 